PROCEEDINGS OF THE

AMERICAN MATHEMATICAL SOCIETY

Volume 131, Number 5, Pages 1369-1372

S 0002-9939(02)06797-7

Article electronically published on October 1, 2002

\title{
KODAIRA DIMENSION OF SYMMETRIC POWERS
}

\author{
DONU ARAPURA AND SVIATOSLAV ARCHAVA \\ (Communicated by Michael Stillman)
}

\begin{abstract}
We compute the plurigenera and the Kodaira dimension of the $d$ th symmetric power $S^{d} X$ of a smooth projective variety $X$. As an application we obtain genus estimates for the curves lying on $X$.
\end{abstract}

We work over the complex numbers. When $X$ is a smooth projective curve of genus $g$, elementary arguments show that the $d$ th symmetric power $S^{d} X$ is uniruled as soon as $d>g$, and therefore that the plurigenera vanish. When the dimension of $X$ is greater than one, the situation is quite different. Using ideas of Roitman Ro1, Ro2] and Reid [Re, we prove:

Theorem 1. Let $X$ be smooth projective variety with $n=\operatorname{dim} X>1$. Let $\Sigma_{d}$ be a desingularization of $S^{d} X$. Then there are isomorphisms

$$
S^{d} H^{0}\left(X, \omega_{X}^{\otimes m}\right) \cong H^{0}\left(\Sigma_{d}, \omega_{\Sigma_{d}}^{\otimes m}\right)
$$

whenever $m n$ is even.

Corollary 1. With the previous assumptions, the mth plurigenus

$$
P_{m}\left(\Sigma_{d}\right)=\left(\begin{array}{c}
d+P_{m}(X)-1 \\
d
\end{array}\right)
$$

whenever $m n$ is even. The Kodaira dimension $\kappa\left(\Sigma_{d}\right)=d \kappa(X)$.

Proof. The first formula is an immediate consequence of the theorem. It implies that

$$
P_{m}\left(\Sigma_{d}\right)=O\left(P_{m}(X)^{d}\right)=O\left(m^{d \kappa(X)}\right)
$$

which yields the second formula.

(D. Huybrechts pointed out to us that these statements were already known in the case $\operatorname{dim} X=2$ and were used to compute the Kodaira dimension of the Hilbert scheme of $d$ points on $X$; see $\underline{\mathrm{HL}}$, theorem 11.1.2.)

Recall that a projective variety $Z$ is uniruled provided there exists a variety $Z^{\prime}$ and dominant rational map $Z^{\prime} \times \mathbb{P}^{1} \rightarrow Z$ which is nontrivial on the second factor. The reference $[\mathrm{K}]$ is more than adequate for standard properties of uniruled varieties.

Corollary 2. If $X$ has nonnegative Kodaira dimension, then $S^{d} X$ is not uniruled for any $d$.

Received by the editors September 1, 2000 and, in revised form, January 9, 2002.

2000 Mathematics Subject Classification. Primary 14J40, 14E08.

The authors were partially supported by the NSF. 
Proof. Since uniruledness is a birational property, it is enough to observe that $\Sigma_{d}$ is not uniruled because it has nonnegative Kodaira dimension.

The most interesting corollaries involve genus estimates for curves lying on $X$. The phrase " $d$ general points of $X$ lie on an irreducible curve with genus $g$ normalization" will mean that there is an irreducible quasiprojective family $\mathcal{C} \rightarrow T$ of smooth projective genus $g$ curves and a morphism $\mathcal{C} \rightarrow X$ which is a generically one-to-one on the fibers $\mathcal{C}_{t}$ and such that the morphism from the relative symmetric power

$$
\mathcal{S}^{d} \mathcal{C}:=\mathcal{C} \times_{T} \mathcal{C} \times_{T} \ldots \mathcal{C} / S_{d}
$$

to $S^{d} X$ is dominant.

Corollary 3. Suppose that the Kodaira dimension of $X$ is nonnegative and that $d$ general points lie on an irreducible curve with genus $g$ normalization. Then $g \geq d$.

Proof. Assume the contrary that $g<d$, and let $\mathcal{C} \rightarrow T$ be the corresponding family. Then each fiber $\mathcal{S}^{d} \mathcal{C}_{t}$ is a projective space bundle over the Jacobian $J\left(\mathcal{C}_{t}\right)$ by AbelJacobi; in particular, it is uniruled. Therefore $\mathcal{S}^{d} \mathcal{C}$ and hence $S^{d} X$ are uniruled, but this contradicts the previous corollary.

Corollary 4. Suppose that $X$ has general type and that $d$ general points lie on an irreducible curve with genus $g$ normalization. Then $g>d$.

Proof. We assume that $g \leq d$ for some family $\mathcal{C} \rightarrow T$. By the previous corollary, we may suppose that $g=d$. Denote the maps $\mathcal{S}^{d} \mathcal{C} \rightarrow S^{d} X$ and $\mathcal{S}^{d} \mathcal{C} \rightarrow T$ by $p$ and $\pi$, respectively. The map $p$ is dominant and generically injective on the fibers of $\pi$. If a general fiber $p^{-1}(Z)$ has positive dimension, then an irreducible hyperplane section $H \subset T$ meets $\pi\left(p^{-1}(Z)\right)$. Therefore $\mathcal{S}^{d} \mathcal{C} \times_{T} H \rightarrow S^{d} X$ is still dominant, and we may replace $T$ by $H$ and $\mathcal{S}^{d} \mathcal{C}$ by the fiber product. By continuing in this way, we can assume that $p$ is generically finite. Choose a desingularization $\bar{T}$ of a compactification of $T$, and a nonsingular compactification of $S$ of $\mathcal{S}^{d} \mathcal{C} \times_{T} \bar{T}$ such that $p$ extends to a morphism of $S$ to a desingularization $\Sigma_{d}$ of $S^{d} X$. We then have $\kappa\left(\Sigma_{d}\right) \leq \kappa(S)$ which implies that $S$ has general type. On the other hand the general fiber of $S \rightarrow \bar{T}$ is $\mathcal{S}^{d} \mathcal{C}_{t}$ is birational to an Abelian variety by the theorems of Abel and Jacobi. This implies that

$$
\kappa(S) \leq \operatorname{dim} \bar{T}+\kappa\left(\mathcal{S}^{d} \mathcal{C}_{t}\right)=\operatorname{dim} \bar{T}<\operatorname{dim} S
$$

by [Mo, 2.3], but this is impossible since $S$ has general type.

\section{Proof of the MAIN THEOREM}

Recall $[\mathrm{Re}]$ that a variety $Y$ has canonical singularities provided that

(1) $Y$ is normal.

(2) $\omega_{Y}^{[r]}:=\left(\omega_{Y}^{\otimes r}\right)^{* *}$ is locally free for some $r>0$, where $\omega_{Y}=\left(\Omega_{Y}^{\operatorname{dim} Y}\right)^{* *}$.

(3) If $f: Y^{\prime} \rightarrow Y$ is a resolution of singularities, then $f_{*} \omega_{Y^{\prime}}^{\otimes r}=\omega_{Y}^{[r]}$.

The smallest such $r$ is called the index. If $Y$ is canonical, then the third condition holds for all $r \geq 1[\mathrm{Re}, 1.3]$. Thus the index is the smallest $r$ for which $\omega_{Y}^{[r]}$ is locally free. It is enough to test the last condition for a particular resolution of singularities. This condition is equivalent to a more widely used condition involving pullbacks of canonical divisors. 
Lemma 1. Let $Z$ be a smooth variety on which a finite group $G$ acts. Let $Y=Z / G$. If $Y$ has canonical singularities of index dividing $r$, then

$$
H^{0}\left(Y, \omega_{Y}^{[r]}\right) \subseteq H^{0}\left(Z, \omega_{Z}^{\otimes r}\right)^{G} .
$$

If the fix point locus has codimension greater than one, equality holds.

Proof. Construct a commutative diagram

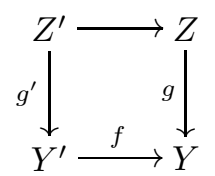

where $Y^{\prime} \rightarrow Y$ is a desingularization, and $Z^{\prime}$ is a $G$-equivariant desingularization of the fiber product. Then there are inclusions

$$
\omega_{Y}^{[r]}=f_{*} \omega_{Y^{\prime}}^{\otimes r} \subseteq\left(f \circ g^{\prime}\right)_{*}\left(\omega_{Z^{\prime}}^{\otimes r}\right)^{G}=g_{*}\left(\omega_{Z}^{\otimes r}\right)^{G} .
$$

This implies the first part of the lemma. The inclusion $\omega_{Y}^{[r]} \subseteq g_{*}\left(\omega_{Z}^{\otimes r}\right)^{G}$ is an equality on the complement of the fixed point locus. Since $\omega_{Y}^{[r]}$ is locally free (hence reflexive) and $g_{*}\left(\omega_{Z}^{\otimes r}\right)^{G}$ is torsion free, the second statement follows.

Proposition 1. Let $X$ be a smooth variety of dimension $n>1$. Then $S^{d} X$ has canonical singularities of index 1 if $n$ is even, and canonical singularities of index at most 2 if $n$ is odd.

Proof. As the result is local analytic for $X$, we may replace it by $\mathbb{C}^{n}$. Consider the action of the symmetric group $S_{d}$ on $\mathbb{C}^{n d}=\mathbb{C}^{n} \times \ldots \times \mathbb{C}^{n}$ by permutation of factors, and let $h: S_{d} \rightarrow G L_{n d}(\mathbb{C})$ be the corresponding homomorphism. This action is equivalent to a direct sum of $n$ copies of the standard representation $\mathbb{C}^{d}$ where $S_{d}$ acts via permutation matrices. Therefore $h\left(S_{d}\right)$ does not contain any quasi-reflections (because $n>1$ ) and $\operatorname{det}(h(\sigma))=\operatorname{sign}(\sigma)^{n}$. When $n$ is even, $h\left(S_{d}\right) \subset S L_{n d}(\mathbb{C})$. This implies that $S^{d} X=\mathbb{C}^{n d} / S_{d}$ is Gorenstein by [W], and therefore canonical of index one [Re, 1.8].

The case when $n$ is odd is more laborious. For any element $\sigma \in S_{d}$ of order $r$, define $S(\sigma)$ as follows: choose a primitive $r$ th root of unity $\epsilon$ and express the eigenvalues of $h(\sigma)$ as $\lambda_{i}=\epsilon^{a_{i}}$ where $0 \leq a_{i}<r$, set $S(\sigma)=\sum a_{i}$. By [Re 3.1], to prove that $S^{d} X$ is canonical it will suffice to verify that $S(\sigma) \geq r$ for every element $\sigma$ of order $r$. Let $\mathbb{C}^{d}$ be the permutation representation of $S_{d}$. If $e_{1}, \ldots, e_{d}$ is the standard basis then $\sigma \cdot e_{i}=e_{\sigma(i)}$. If $\epsilon$ is a primitive $r$ th root of unity, then it is easy to see that the eigenvectors of the cycle $\sigma=(12 \ldots r)$ acting on $\mathbb{C}^{d}$ are $e_{1}+\epsilon^{i} e_{2}+\ldots+\epsilon^{(r-1) i} e_{r}$ and $e_{r+1}, \ldots, e_{d}$. Therefore the nonunit eigenvalues are $\epsilon, \ldots, \epsilon^{r-1}$ and these occur with multiplicity one. Hence $S(\sigma)=n r(r-1) / 2 \geq r$ as required. The general case is similar. Let $\sigma$ be a permutation of order $r$ and $\epsilon$ as before. Write $\sigma$ as a product of disjoint cycles of length $r_{i}$. Therefore $r$ is the least common multiple of the $r_{i}$, and let $r_{i}^{\prime}=r / r_{i}$. A list (with possible repetitions) of the nonunit eigenvalues of $\sigma$ acting on $\mathbb{C}^{d}$ is

$$
\epsilon^{r_{1}^{\prime}}, \ldots, \epsilon^{r_{1}^{\prime}\left(r_{1}-1\right)}, \epsilon^{r_{2}^{\prime}}, \ldots, \epsilon^{r_{2}^{\prime}\left(r_{2}-1\right)}, \ldots
$$

Therefore

$$
S(\sigma)=\frac{n}{2}\left[r_{1}^{\prime} r_{1}\left(r_{1}-1\right)+r_{2}^{\prime} r_{2}\left(r_{2}-1\right)+\ldots\right] \geq r
$$


and this proves that $S^{d} X$ is canonical. It remains to check that the index is at most 2. For this it suffices to observe that if $x_{i}$ are coordinates on $\mathbb{C}^{\text {nd }}$, then

$$
\left(d x_{1} \wedge \ldots \wedge d x_{n d}\right)^{\otimes 2}
$$

is $S_{d}$ invariant. This determines a generator of $\omega_{S^{d} X}^{[2]}(X)$, which shows that this module is free.

Proof of the main theorem. Let $m$ be an integer such that $m n$ is even (hence a multiple of the index of $\left.S^{d} X\right)$. Then

$$
H^{0}\left(\omega_{\Sigma_{d}}^{\otimes m}\right)=H^{0}\left(\omega_{S^{d} X}^{[m]}\right)=H^{0}\left(\omega_{X^{d}}^{\otimes m}\right)^{S_{d}}
$$

By Künneth's formula, this equals

$$
\left[H^{0}\left(\omega_{X}^{\otimes m}\right) \otimes \ldots \otimes H^{0}\left(\omega_{X}^{\otimes m}\right)\right]^{S_{d}}=S^{d} H^{0}\left(\omega_{X}^{\otimes m}\right) .
$$

\section{REFERENCES}

[HL] D. Huybrechts, M. Lehn, The geometry of Moduli Spaces of Sheaves Aspects of Mathematics E31, Friedr. Vieweg \& Sohn, Braunschweig (1997) MR 98g:14012

[K] J. Kollár, Rational curves on algebraic varieties Springer-Verlag (1996) MR 98c:14001

[Mo] S. Mori, Classification of higher dimensional varieties Algebraic Geometry Bowdoin 1985, AMS (1987) MR 89a:14040

[M] D. Mumford, Rational equivalence of 0-cycles on surfaces J. Math. Kyoto Univ. 9 (1969) MR 40:2673

[Re] M. Reid, Canonical 3-folds, Journeés Géometrie Algébrique, Sijthoff and Noordorff (1980) MR 82i: 14025

[Ro1] A. A. Roitman On $\Gamma$-equivalence of zero-dimensional cycles USSR Sbornik 86 (1971) MR 44:6701

[Ro2] A. A. Roitman Rational equivalence of 0-dimensional cycles USSR Sbornik 89 (1972) MR 48:6109

[W] K. Watanabe, Certain invariant subrings are Gorenstein II Osaka Math. J. 11 (1974) MR 50:7124

Department of Mathematics, Purdue University, West Lafayette, Indiana 47907

E-mail address: arapura@math.purdue.edu

MPI für Mathematik, Vivatgasse 7, D-53111, Bonn, Germany 